\title{
First-principles study of the mobility of $\mathrm{SrTiO}_{3}$
}

\author{
Burak Himmetoglu, Anderson Janotti, Hartwin Peelaers, Audrius Alkauskas, and Chris G. Van de Walle \\ Materials Department, University of California, Santa Barbara, CA 93106-5050
}

(Dated: May 27, 2022)

\begin{abstract}
We investigate the electronic and vibrational spectra of $\mathrm{SrTiO}_{3}$, as well as the coupling between them, using first-principles calculations. We compute electron-phonon scattering rates for the three lowest-energy conduction bands and use Boltzmann transport theory to calculate the room-temperature mobility of $\mathrm{SrTiO}_{3}$. The results agree with experiment and highlight the strong impact of longitudinal optical phonon scattering. Our analysis provides important insights into the key factors that determine room temperature mobility, such as the number of conduction bands and the nature and frequencies of longitudinal phonons. Such insights provide routes to engineering materials with enhanced mobilities.
\end{abstract}

There is great interest in using $\mathrm{SrTiO}_{3}$ (STO) as a wide-band-gap semiconductor in novel electronic devices. Thanks to recent progress in epitaxial growth of STO, 1 $n$-doped films have been achieved with carrier mobility as high as $53,000 \mathrm{~cm}^{2} \mathrm{~V}^{-1} \mathrm{~s}^{-1}$ at $T=2 \mathrm{~K} .^{[2}$ However, roomtemperature mobilities are orders of magnitude smaller, around a few $\mathrm{cm}^{2} \mathrm{~V}^{-1} \mathrm{~s}^{-1}$, potentially forming a significant limitation in electronic device applications ${ }^{1}$ and lending urgency to the investigation of the key material parameters that affect electron transport. Electron mobility and other transport properties depend crucially on the conduction-band structure of STO, which has already been investigated in a number of studies. ${ }^{[-5}$ Still, a clear understanding of the transport properties of STO has not yet been established.

Transport properties, conduction mechanisms, and the dependence of mobility on temperature and electron density of STO have been investigated both in single crysta ${ }^{6}$ and epitaxially grown samples ${ }^{7}$ Recent experiments based on the measurement of Shubnikov de Haas oscillations ${ }^{314}$ in STO thin films yielded effective band masses that are significantly larger than those obtained from band-structure calculations. ${ }^{[8}$ The mass enhancement was attributed to the strong electron-phonon coupling in STO 3 The effect of electron-phonon interactions has also been discussed in relation to electron mobility. Typically, the rapid decrease of the electron mobility with increasing temperature has been associated with scattering of conduction electrons by polar optical phonon modes. ${ }^{910}$ Consequences of electron-phonon scattering on the effective mass of electrons in the conduction bands have been studied by analyzing the spectral weight of the experimental optical conductivity ${ }^{11}$ and within the context of large-polaron models $\frac{12}{12}$ More recently, the effect of electron-phonon scattering over a wide temperature range has been investigated for thin films. ${ }^{[7}$ An analysis based on phenomenological models is consistent with longitudinal optical ( $\mathrm{LO}$ ) phonon scattering determining the room-temperature mobility, while at lower temperatures (between 2 and $200 \mathrm{~K}$ ), transverse optical (TO) modes were also found to be important.

Here we investigate the vibrational and electronic spectra of STO, as well as their coupling. We find that polar optical mode scattering leads to low mobilities at room temperature. Our analysis provide insights into the mechanisms that could potentially be used to tune the scattering, for instance by nanostructuring, by using epitaxial strain (which was already discussed in the context of effective masses ${ }^{8}$ ), or by replacing $\mathrm{Ti}$ with a heavier transition metal to increase spin-orbit splittings, leading to possible enhancement of mobility.

Very few first-principles calculations of mobilities including electron-phonon interactions have appeared in the literature, for other materials ${ }^{13}[15$ Obtaining converged scattering rates and transport integrals has been very challenging. Our present work is based on accurate interpolation schemes and enabled by the use of an analytical model for electron-phonon coupling, which we explicitly justify. These developments allow us to obtain converged results at reasonable computational cost.

Our calculations are performed with the plane-wave self-consistent field (PWSCF) code of the Quantum ESPRESSO package, $\frac{16}{16}$ using ultra-soft pseudopotentials. ${ }^{17}$ We use the local density approximation (LDA) with the Perdew-Zunger parametrization $\frac{\sqrt[18]{ }}{}$ we will verify that the use of the LDA provides adequate accuracy for the quantities of interest in this work. Plane-wave basis sets are used to expand wavefunctions and charge densities, with kinetic energy cutoffs of 50 Ry and 600 Ry, respectively. The phonon spectrum is calculated using density functional perturbation theory (DFPT) as implemented in Quantum ESPRESSO 19 Brillouin-zone (BZ) integrations are performed on a $8 \times 8 \times 8$ special $k$-point grid ${ }^{20}$ for self-consistent field calculations. The phonon spectrum is calculated on a $4 \times 4 \times 4$ special point grid, and interpolated along lines connecting special points within the BZ. The splitting of longitudinal and transverse optical modes at $\Gamma$ has been taken into account with the method of Born and Huang. 21 The Fermi integral for the calculation of the conductivity tensor is performed by the BoltzTrap code.22

We consider the cubic phase of $\mathrm{SrTiO}_{3}$, which is the stable phase at room temperature. The optimized lattice parameter is $a_{0}=3.86 \AA$, about $1 \%$ smaller than experiment ${ }^{23}$ as expected from the LDA functional. The LDA band structure is shown in Fig. 1 . The valence bands are derived from $\mathrm{O} p$ orbitals, while the lowest three conduction bands are derived from the $\mathrm{Ti} 3 d \mathrm{t}_{2 g}$ orbitals $\left(d_{x y}\right.$, 
$d_{z x}$ and $\left.d_{y z}\right)$. Due to the localized nature of these $\mathrm{Ti}$ $3 d$ orbitals, the conduction bands have low dispersion, leading to high effective masses. As expected, LDA underestimates the band gap, producing an indirect $\mathrm{R}-\Gamma$ gap of $1.84 \mathrm{eV}$ and a direct $\Gamma-\Gamma$ gap of $2.15 \mathrm{eV}$, compared to the experimental values of $3.25 \mathrm{eV}$ and $3.75 \mathrm{eV}$, respectively ${ }^{24}$ In all other respects, however, LDA produces a reliable band structure. In particular, the shape of the conduction bands and the effective masses are in good agreement with more accurate calculations ${ }^{8}$ using the hybrid functional of Heyd, Scuseria and Ernzerhof (HSE) $\stackrel{25}{2}$ as can be seen from Table. I.

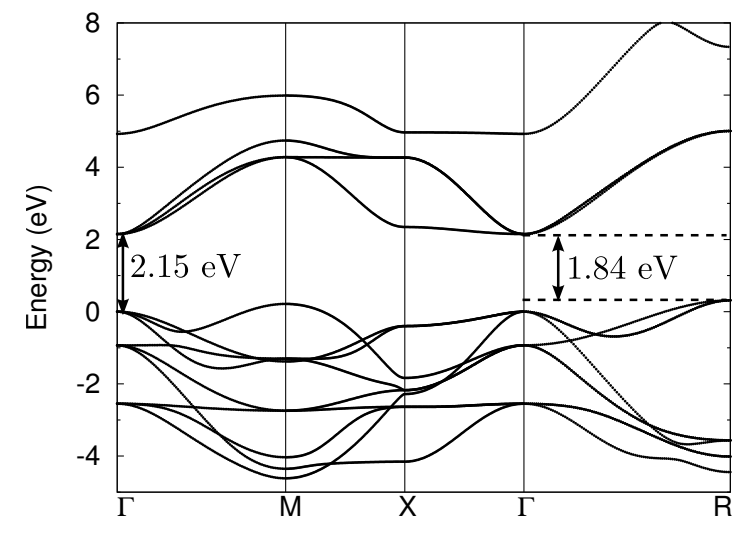

FIG. 1. Calculated band structure of cubic $\mathrm{SrTiO}_{3}$ using the LDA functional.

TABLE I. Calculated band masses (in units of electron mass) along the $\Gamma-\mathrm{X}$ and $\Gamma-\mathrm{M}$ directions, based on the LDA and the HSE hybrid functional. HSE values were taken from Ref. 8 .

\begin{tabular}{|c|c|c|c|c|}
\hline & \multicolumn{2}{|c|}{$\overline{\Gamma-X}$} & \multicolumn{2}{|c|}{$\overline{\Gamma-\mathrm{M}}$} \\
\hline & LDA & HSE & LDA & HSE \\
\hline$\overline{m_{1}}$ & 6.11 & 6.10 & 0.77 & 0.85 \\
\hline$m_{2}$ & 0.35 & 0.39 & 0.56 & 0.64 \\
\hline$m_{3}$ & 0.35 & 0.39 & 0.35 & 0.39 \\
\hline
\end{tabular}

The phonon spectrum obtained from DFPT is shown in Fig. 2. Table II lists the calculated optical phonon frequencies at $\Gamma$, showing good agreement with experimental results. The cubic phase of STO exhibits soft modes, as indicated by the presence of negative frequencies in the phonon spectrum (see Fig. 2). These soft modes are related to structural instabilities associated with the distortions that lead to the low-temperature tetragonal phase of STO. The cubic phase is stabilized at finite temperature through strong anharmonic effects, which are not included in our calculations based on the harmonic approximation. These soft modes are not expected to be important at room temperature and for the types of electron-phonon scattering mechanisms that are considered in the present study.

Since the conduction-band minimum is located at $\Gamma$, the Fermi surface for $n$-doped STO is always $\Gamma$-centered. Moreover, for experimentally achievable doping levels

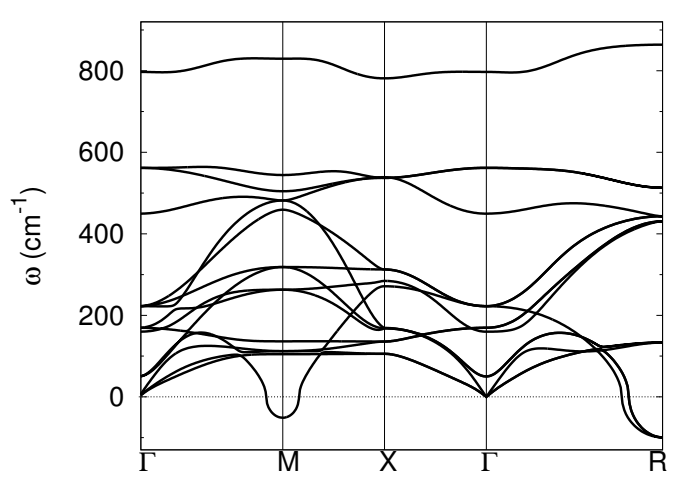

FIG. 2. Calculated phonon spectrum of $\mathrm{SrTiO}_{3}$ using the LDA functional.

TABLE II. Calculated and experimental longitudinal optical (LO) and transverse optical (TO) phonon frequencies at the $\Gamma$ point, in units of $\mathrm{cm}^{-1}$.

\begin{tabular}{cccc}
\hline \hline & $\omega_{\text {LO }}$ & & \multicolumn{2}{c}{$\omega_{\text {TO }}$} \\
LDA & Exp. & LDA & Exp. \\
\hline 160 & $171^{a}$ & 50 & $42^{a}, 91^{b}$ \\
449 & $474^{a}$ & 170 & $175^{a}, 170^{b}$ \\
797 & $795^{a}$ & 222 & $265^{a}$ \\
& & 562 & $545^{a}, 547^{b}$ \\
\hline \hline
\end{tabular}

a Ref. 26.

${ }^{b}$ Ref. 27

$\left(n<10^{21} \mathrm{~cm}^{-3}\right)$, the Fermi surface covers only a small portion of the BZ. The smallness of the Fermi surface then requires a very fine sampling of the BZ. In order to achieve the fine sampling, we have constructed maximally localized Wannier functions $\sqrt{28}$ and interpolated the band structure of STO to a grid of $50 \times 50 \times 50 k$-points. The Fermi surface resulting from the Wannier interpolation for $n=10^{20} \mathrm{~cm}^{-3}$ is shown in Fig. 3. As a result, (a)

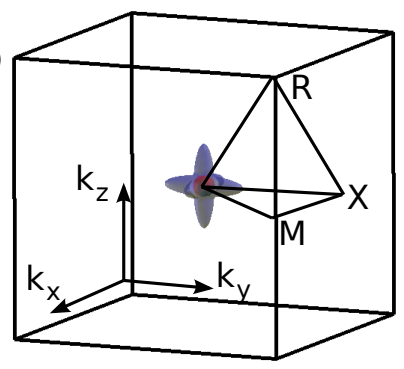

(b)

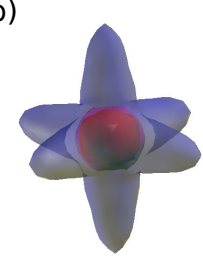

FIG. 3. (color online) (a) Fermi surface of $\mathrm{SrTiO}_{3}$ for an electron concentration $n=10^{20} \mathrm{~cm}^{-3}$, shown in the Brillouin zone, with high-symmetry points indicated. (b) Magnified view of the Fermi surface. There are two low-mass bands with spherical shape (red) and one high-mass band with an ellipsoidal shape (blue). 
only phonons with very small wavevectors are able to scatter electrons. In other words, only long-wavelength phonons contribute to the finite scattering time of the electrons. Anharmonic effects that lead to stabilization of soft modes could introduce additional electron-phonon scattering channels. These could be important around the temperature at which the tetragonal-to-cubic phase transition occurs (110 K, Ref. 29), but this is not the focus of our present study.

For polar crystals, electron-phonon scattering for phonons with small wavevectors $(\mathbf{q})$ is dominated by LO mode scattering. $\stackrel{30}{\text { It }}$ can be analytically shown that LO mode scattering results in electron-phonon coupling matrix elements that have a $1 / q$ dependence. Therefore, for electrons in conduction bands near $\Gamma$ at temperatures where LO modes have large occupations, this scattering channel dominates. In this study we use the Fröhlich model ${ }^{30}$ which provides a simple but accurate description of LO mode scattering with the electron-phonon coupling matrix elements given by

$$
g_{\mathbf{q} \nu}=\sqrt{\frac{e^{2} \hbar \omega_{\nu}}{2 \epsilon_{0} V_{\text {cell }} q^{2}}} \sqrt{\frac{1}{\epsilon_{\infty}}-\frac{1}{\epsilon}}
$$

where $\omega_{\nu}$ are LO phonon frequencies, $\epsilon_{0}$ is the vacuum permittivity, $V_{\text {cell }}$ is the unit cell volume, and $\epsilon_{\infty}$ and $\epsilon$ are the electronic and static dielectric constants, respectively. In principle, longitudinal acoustic (LA) and TO modes also contribute to electron-phonon scattering. However, for small wavevectors and at temperatures where LO mode occupancy is sizable, their contribution is much smaller than that of LO modes $\sqrt[30]{ }$

Electrons in a state $\psi_{n \mathbf{k}}$ experience a scattering rate due to electron-phonon interactions that can be obtained from Fermi's Golden rule as: ${ }^{30}$

$$
\begin{gathered}
\tau_{n \mathbf{k}}^{-1}=\frac{2 \pi}{\hbar} \sum_{\mathbf{q} \nu, m}\left|g_{\mathbf{q} \nu}(\mathbf{k}, n, m)\right|^{2}\left(1-\hat{v}_{n \mathbf{k}} \cdot \hat{v}_{m \mathbf{k}+\mathbf{q}}\right) \times \\
\left\{\left(n_{\mathbf{q} \nu}+f_{m, \mathbf{k}+\mathbf{q}}\right) \delta\left(\epsilon_{m, \mathbf{k}+\mathbf{q}}-\epsilon_{n \mathbf{k}}-\hbar \omega_{\mathbf{q} \nu}\right)\right. \\
\left.+\left(1+n_{\mathbf{q} \nu}-f_{m, \mathbf{k}+\mathbf{q}}\right) \delta\left(\epsilon_{m, \mathbf{k}+\mathbf{q}}-\epsilon_{n \mathbf{k}}+\hbar \omega_{\mathbf{q} \nu}\right)\right\}
\end{gathered}
$$

where $g_{\mathbf{q} \nu}(\mathbf{k}, n, m)$ are electron-phonon coupling matrix elements, the velocities $\hat{v}_{n \mathbf{k}}$ are defined below, and $n_{\mathbf{q} \nu}$ and $f_{m, \mathbf{k}+\mathbf{q}}$ are phonon and electron occupation factors described by Bose-Einstein and Fermi-Dirac distributions respectively. The delta functions ensure energy conservation. Since the electrons all reside in the vicinity of the $\Gamma$ point (Fig. 3), only phonons with small wavevectors can satisfy the energy conservation criterion between electronic states. The Fröhlich coupling given by Eq. (1) has no dependence on the electron wavevector $\mathbf{k}$; ignoring the $\mathbf{k}$-dependence of the electron-phonon matrix element is a good approximation since the Fermi surface occupies only a small portion of the BZ. A further approximation inherent to the Fröhlich model is the neglect of the dependence of the electron-phonon coupling matrices on the band indices, resulting in all scattering rates between the different conduction bands being equivalent.
The band velocities in Eq. (2) are defined as

$$
\mathbf{v}_{n \mathbf{k}}=\frac{1}{\hbar} \frac{\partial \epsilon_{n \mathbf{k}}}{\partial \mathbf{k}}, \hat{v}_{n \mathbf{k}}=\mathbf{v}_{n \mathbf{k}} /\left|\mathbf{v}_{n \mathbf{k}}\right|
$$

The velocity factor $\left(1-\hat{v}_{n \mathbf{k}} \cdot \hat{v}_{m \mathbf{k}+\mathbf{q}}\right)$ in Eq. (2) is added in an ad hoc manner ${ }^{30}$ to include the effect of directionality in the transport scattering rate. While the velocity factor has been shown to be crucial in order to obtain the right temperature dependence of resistivity in simple metals $\stackrel{30}{30}$ we have explicitly checked that it does not produce significant modifications in our calculations for STO; including the factor changes the scattering rate by only $\sim 10 \%$.

The electron-phonon matrix elements in Eq. (1) can be evaluated using the calculated LO phonon mode frequencies shown in Table II and the dielectric constants. The optical phonons have a rather flat spectrum (Fig. 22, therefore their dispersion can be ignored. Since $\epsilon \gg$ $\epsilon_{\infty}, 31$ one can ignore the $1 / \epsilon$ term. The calculated dielectric constant $\epsilon_{\infty}=6.34$ is in reasonable agreement with the experimental value of $\epsilon_{\infty} \simeq 5.59 \stackrel{31}{\text { Evaluating }}$ the constants in Eq. (1), we can rewrite it as

$$
g_{\mathbf{q} \nu}=\frac{C_{\nu}}{\left(q \cdot a_{0} / 2 \pi\right)}
$$

The constants $C_{\nu}$ are calculated as $C_{1}=0.0024 \mathrm{eV}^{2}$, $C_{2}=0.0066 \mathrm{eV}^{2}$, and $C_{3}=0.012 \mathrm{eV}^{2}$, where $C_{\nu}$ are indexed in order of increasing frequency, as listed in Table I

Using the electron-phonon coupling matrices of Eq. (4), we obtain the electron-phonon scattering rates from Eq. (2) for the three conduction bands with effective masses listed in Table $\mathrm{I}$. We performed the calculations at $300 \mathrm{~K}$, using a $50 \times 50 \times 50$ grid of $q$-points. The energy-conserving delta functions are replaced by Gaussians with a width of $0.05 \mathrm{eV}$. The choice of the $q$-point grid and smearing yields an accuracy of $0.02 \mathrm{fs}$ for the calculated scattering times. The resulting scattering rates are shown in Fig. 4 along the high-symmetry directions $\Gamma-\mathrm{X}$ and $\Gamma$-M.

Electrons with heavy masses (mass $m_{1}$ ) have the lowest scattering rate in most of the BZ, while lighter electrons are scattered more effectively. This result can be understood by investigating the effect of atomic displacements associated with polar optical modes on the band structure. We show in Fig. 5 the displacements of atoms for the highest frequency polar optical mode $\left(\omega_{1}=797\right.$ $\left.\mathrm{cm}^{-1}\right)$. The relative displacements of the atoms are obtained from the eigenvectors of the dynamical matrix at $\Gamma$. In cubic symmetry, the $d_{x y}$, and $d_{y z}$, and $d_{z x}$ orbitals of $\mathrm{Ti}$ are in principle equivalent. But when a particular LO mode is chosen. e.g., pointing along $x$, as in Fig. 5, the symmetry is broken and the heavy band becomes the one derived from the $d_{y z}$ orbital, while the $d_{x y^{-}}$and $d_{z x^{-}}$-derived bands are lighter. The O-atom displacements corresponding to this mode bring the $\mathrm{O} p$ orbitals closer to $d_{x y}$ and $d_{z x}$, resulting in strong repulsion between filled $\mathrm{O} p$ shells and conduction electrons 


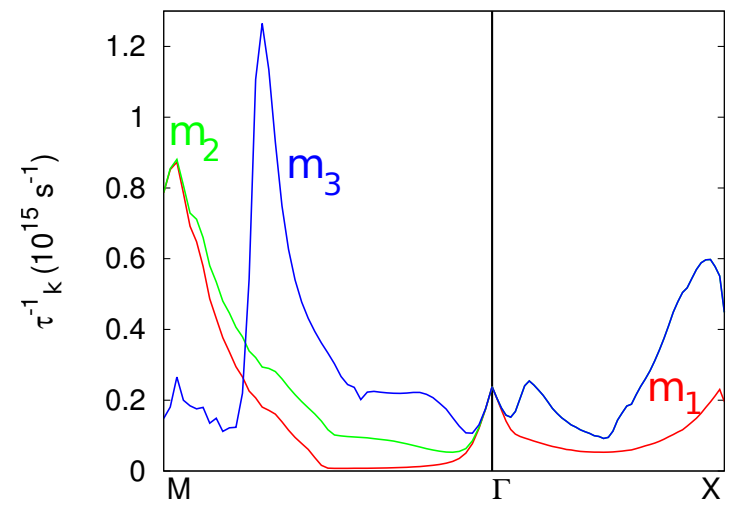

FIG. 4. (color online) Electron-phonon scattering rates for electrons with a given momentum between $\Gamma$ and $M$ or between $\Gamma$ and $\mathrm{X}$, for the three conduction bands, at $300 \mathrm{~K}$ and for $n=10^{20} \mathrm{~cm}^{-3}$.

occupying these two $\mathrm{t}_{2 g}$ bands. The strong repulsion results in a scattering rate which is larger for the low-mass band (Fig. 4). On the other hand, $\mathrm{O} p$ and $d_{y z}$ orbitals do not approach each other during displacements arising from the polar optical mode, and thus the scattering rate is smaller for the heavy band (Fig. 4).
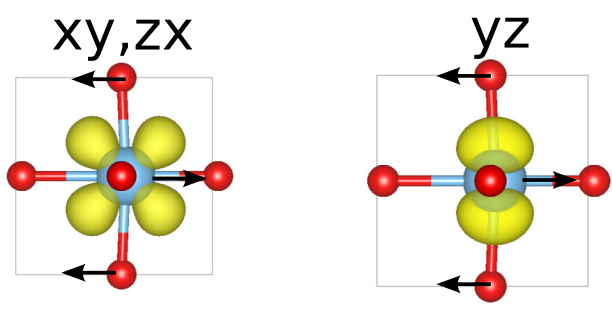

FIG. 5. (color online) Atomic displacements corresponding to the highest frequency LO mode and $t_{2 g}$ orbital isosurfaces representing the conduction bands.

The actual mobility of electrons is determined both by the scattering rates and the effective masses. In order to obtain the room-temperature mobility, we compute the conductivity tensor within Boltzmann transport theory given by

$$
\sigma_{\alpha \beta}=\frac{2 e^{2}}{V_{\text {cell }}} \sum_{n, \mathbf{k}} \tau_{n \mathbf{k}}\left(-\frac{\partial f_{n \mathbf{k}}}{\partial \epsilon_{n \mathbf{k}}}\right) v_{n \mathbf{k}, \alpha} v_{n \mathbf{k}, \beta}
$$

where $\alpha$ and $\beta$ are Cartesian indices. Since STO is cubic, the conductivity tensor is diagonal with equal entries. To evaluate Eq. (5), we assume the scattering time $\tau_{n \mathbf{k}}$ is given by its value at $\Gamma$. This approximation is again justified by the smallness of the region of the BZ occupied by the Fermi surface. We compute the scattering rates and electron mobilities using $\mu=\sigma / n e$ at $300 \mathrm{~K}$ for a range of carrier densities between $10^{18} \mathrm{~cm}^{-3}$ and $10^{21}$ $\mathrm{cm}^{-3}$. We find mobilities between 7 and $18 \mathrm{~cm}^{2} / \mathrm{V} / \mathrm{s}$, in good agreement with experimental values $\frac{1121619110}{10}$

The insights provided by our calculations allow us to suggest several routes to achieving enhanced mobilities by engineering materials or using alternative compounds. (1) Equation (2) indicates that electron-phonon scattering is ineffective when the LO phonon energy does not match the energy difference between the initial $\left(\epsilon_{n \mathbf{k}}\right)$ and the final band energies $\left(\epsilon_{m, \mathbf{k}+\mathbf{q}}\right)$. Splitting the bands by an amount that exceeds the LO phonon frequencies will reduce the scattering rates and result in an increase in electron conductivity. This is due to the fact that the number of available final states where an electron is scattered by a phonon becomes smaller. Given that the maximum LO phonon frequency is around $0.1 \mathrm{eV}$, such splitting can be achieved by replacing Ti with a heavier transition metal that would yield a splitting due to stronger spin-orbit coupling.

(2) Nanostructuring can be used to exploit quantum confinement and discretize the electronic spectrum with energy differences that do not match phonon frequencies. Similar physics has been discussed in the context of quantum dots, where it is known as the phonon-bottleneck effect ${ }^{32}$

(3) The scattering rate can be reduced by decreasing the number of (nearly) degenerate conduction bands. Perovskites with a conduction band derived from a single orbital, such as $\mathrm{BaSnO}_{3}$, display much higher roomtemperature mobilities ${ }^{[33}$ Part of this effect is of course due to the fact that in $\mathrm{BaSnO}_{3}$ the conduction band is derived from $s$ states, providing a smaller effective electron mass; however, the reduction in the number of scattering channels for electrons via optical phonons also contributes significantly to the enhancement in mobility. In order to demonstrate this effect, we have evaluated the scattering rates in Eq. (2) using only a single band (with mass $m_{1}$ ). We find that the reduction of the number of conduction bands from 3 to 1 (assuming a fixed Fermilevel position) yields $\tau_{3 \text { bands }}^{-1} / \tau_{1 \text { band }}^{-1} \simeq 2.05$ at $\Gamma$.

(4) Strain can be used to modify the shape and degeneracy of conduction bands. In fact, a factor of 2 to 3 increase in mobility was observed under compressive strain in STO at low temperatures 5 Strain modifies the effective masses $\frac{8}{8}$ which by itself can lead to changes in mobility. In addition, strain can break the degeneracy of the three lowest energy conduction bands, which scatter differently. The strain that can be achieved by pseudomorphic growth is probably not large enough to split the conduction bands by an amount larger than the LO phonon frequency, ${ }^{8}$ but it can still lead to modest enhancements in mobility. The three lowest energy conduction bands in STO scatter differently with LO phonons, yielding different scattering rates along the BZ (see Fig. 4). Biaxial strain breaks the degeneracy of the bands at $\Gamma$ and will modify the scattering rates (which in the unstrained case are very similar for the different bands, close to $\Gamma$ ).

(5) Strain also modifies the LO phonon frequencies. As can be seen from Eqs. (1) and 22), the scattering rate of electrons from LO phonons is proportional to the phonon frequency. Tensile strain along the atomic displacements of the LO modes (see Fig. 5) could lower the force con- 
stants and lead to lower frequencies. In addition, lower LO phonon frequencies make it harder for electrons to reach the next available conduction band, which acts as an additional mechanism to reduce scattering. Pseudomorphic growth on specific substrates ${ }^{8}$ could thus be a powerful tool for engineering mobilities.

In conclusion, we have developed a computationally tractable first-principles methodology for calculating mobilities of polar materials. We have explicitly shown that the room-temperature mobility of electrons in STO is determined by longitudinal optical mode scattering, and provided insights into avenues for improving mobility.

This work was supported by the MURI program of the Office of Naval Research, Grant Number N0001412-1-0976. Additional support was provided by ARO (W911-NF-11-1-0232). Computational resources were provided by the Center for Scientific Computing at the CNSI and MRL (an NSF MRSEC, DMR-1121053) (NSF CNS-0960316), and by the Extreme Science and Engineering Discovery Environment (XSEDE), supported by NSF (OCI-1053575 and DMR07-0072N).
1 J. Son, P. Moetakef, B. Jalan, O. Bierwagen, N. J. Wright, R. Engel-Herbert, and S. Stemmer, Nat. Mater. 9, 482 (2010)

2 T. A. Cain, A. P. Kajdos, and S. Stemmer, Appl. Phys. Lett. 102, 182101 (2013)

3 M. Kim, C. Bell, Y. Kozuka, M. Kurita, Y. Hikita, and H. Y. Hwang, Phys. Rev. Lett. 107, 106801 (2011)

${ }^{4}$ S. J. Allen, B. Jalan, S. Lee, D. G. Ouellette, G. Khalsa, J. Jaroszynski, S. Stemmer, and A. H. MacDonald, Phys. Rev. B 88, 045114 (2013)

5 B. Jalan, S. J. Allen, G. E. Beltz, P. Moetakef, and S. Stemmer, Appl. Phys. Lett. 98, 132102 (2011)

6 A. Spinelli, M. A. Torija, C. Liu, C. Jan, and C. Leighton, Phys. Rev. B 81, 155110 (2010)

7 A. Verma, A. P. Kajdos, T. A. Cain, S. Stemmer, and D. Jena, Phys. Rev. Lett. 112, 216601 (2014)

8 A. Janotti, D. Steiauf, and C. G. Van de Walle, Phys. Rev. B 84, 201304 (2011)

9 H. P. R. Frederikse and W. R. Hosler, Phys. Rev. 161, 822 (1967)

10 O. N. Tufte and P. W. Chapman, Phys. Rev. 155, 796 (1967)

11 J. L. M. van Mechelen, D. van der Marel, C. Grimaldi, A. B. Kuzmenko, N. P. Armitage, N. Reyren, H. Hagemann, and I. I. Mazin, Phys. Rev. Lett. 100, 226403 (2008)

12 J. T. Devreese, S. N. Klimin, J. L. M. van Mechelen, and D. van der Marel, Phys. Rev. B 81, 125119 (2010)

13 O. D. Restrepo, K. Varga, and S. T. Pantelides, Appl. Phys. Lett. 94, 212103 (2009)

14 K. Kaasbjerg, K. S. Thygesen, and K. W. Jacobsen, Phys. Rev. B 85, 115317 (2012)

15 K. M. Borysenko, J. T. Mullen, E. A. Barry, S. Paul, Y. G. Semenov, J. M. Zavada, M. Buongiorno-Nardelli, and K. W. Kim, Phys. Rev. B 81, 121412 (2010)

16 P. Giannozzi, S. Baroni, N. Bonini, M. Calandra, R. Car, C. Cavazzoni, D. Ceresoli, G. Chiarotti, M. Cococcioni,
I. Dabo, et al., J. Phys. Condens. Matter 21, 395502 (2009)

17 D. Vanderbilt, Phys. Rev. B 41, 7892 (1990)

18 J. P. Perdew and A. Zunger, Phys. Rev. B 23, 5048 (1981)

19 S. Baroni, S. de Gironcoli, A. Dal Corso, and P. Giannozzi, Rev. Mod. Phys. 73, 515 (2001)

20 M. Methfessel and A. Paxton, Phys. Rev. B 40, 3616 (1989)

21 M. Born and K. Huang, Dynamical theory of crystal lattices (Clarendon Press Oxford, 1954)

22 G. K. H. Madsen and D. J. Singh, Comput. Phys. Commun. 175, 67 (2006)

23 L. Cao, E. Sozontov, and J. Zegenhagen, Phys. Status Solidi A 181, 387 (2000)

${ }^{24}$ K. Van Benthem, C. Elsässer, and R. H. French, J. Appl. Phys. 90, 6156 (2001)

25 J. Heyd, G. E. Scuseria, and M. Ernzerhof, J. Chem. Phys. 118, 8207 (2003)

26 J. L. Servoin, Y. Luspin, and F. Gervais, Phys. Rev. B 22, $5501(1980)$

27 W. G. Stirling, Journal of Physics C: Solid State Physics $\mathbf{5}, 2711(1972)$

28 A. A. Mostofi, J. R. Yates, Y. S. Lee, I. Souza, D. Vanderbilt, and N. Marzari, Comput. Phys. Commun. 178, 685 (2008)

29 R. A. Cowley, W. J. L. Buyers, and G. Dolling, Solid State Communications 7, 181 (1969)

30 J. M. Ziman, Electrons and phonons: the theory of transport phenomena in solids (Oxford University Press, 2001)

31 W. G. Spitzer, R. C. Miller, D. A. Kleinman, and L. E. Howarth, Phys. Rev. 126, 1710 (1962)

32 A. J. Nozik, Annu. Rev. Phys. Chem. 52, 193 (2001)

${ }^{33}$ H. J. Kim, U. Kim, H. M. Kim, T. H. Kim, H. S. Mun, B. Jeon, K. T. Hong, W. Lee, C. Ju, K. H. Kim, and K. Char, Appl. Phys Express 5, 061102 (2012) 